\title{
A Simple and Effective Clustering Algorithm for Multispectral Images Using Space-Filling Curves
}

\author{
Jian ZHANG $^{\dagger a)}$, Nonmember and Sei-ichiro KAMATA ${ }^{\dagger b)}$, Member
}

\begin{abstract}
SUMMARY With the wide usage of multispectral images, a fast efficient multidimensional clustering method becomes not only meaningful but also necessary. In general, to speed up the multidimensional images' analysis, a multidimensional feature vector should be transformed into a lower dimensional space. The Hilbert curve is a continuous one-to-one mapping from $N$-dimensional space to one-dimensional space, and can preserves neighborhood as much as possible. However, because the Hilbert curve is generated by a recurve division process, 'Boundary Effects' will happen, which means data that are close in $N$-dimensional space may not be close in one-dimensional Hilbert order. In this paper, a new efficient approach based on the space-filling curves is proposed for classifying multispectral satellite images. In order to remove 'Boundary Effects' of the Hilbert curve, multiple Hilbert curves, $z$ curves, and the Pseudo-Hilbert curve are used jointly. The proposed method extracts category clusters from one-dimensional data without computing any distance in $N$-dimensional space. Furthermore, multispectral images can be analyzed hierarchically from coarse data distribution to fine data distribution in accordance with different application. The experimental results performed on LANDSAT data have demonstrated that the proposed method is efficient to manage the multispectral images and can be applied easily.

key words: space-filling curves, Euclidean distance, data clustering, multispectral images
\end{abstract}

\section{Introduction}

With development of remote sensing technology, multispectral satellite images have been applied widely in many fields, such as global environments monitoring, natural resource management, geographic information systems and land use planning. In these fields, clustering, as one of the most important techniques for discovering interesting data in the underlying data objects, has been received a lot of research. Currently there exist several clustering algorithms, such as $k$-means [11], overlapping clustering algorithms [12], [13], [19], mixture of Gaussians [14]. However, these algorithms usually use recursive functions to search clusters, and the randomly selected initial values are very sensitive to speed of convergence. These make algorithms complex and and difficult to be put in practice. Especially in multispectral images, each pixel is described by a multidimensional vector, so the computational complexities of algorithms would become very high with increase of dimensionality and clusters. To overcome this problem and speed up the traditional

\footnotetext{
Manuscript received November 7, 2011.

Manuscript revised January 30, 2012.

${ }^{\dagger}$ The authors are with the Graduate School of Information, Production and Systems, Waseda University, Kitakyushu-shi, 8080135 Japan.

a)E-mail: zj_jay@toki.waseda.jp

b) E-mail: kam@waseda.jp

DOI: 10.1587/transinf.E95.D.1749
}

clustering algorithm, generally a technique for reduction of dimensionality is required. Currently, several methods [5], [16], [17] for linear mapping of a multidimensional space have been proposed. Among these methods, principal component analysis (PCA) is most commonly used technique to reduce the dimensionality of the multispectral images. Its most desired property is that the locality of a multidimensional space can be preserved in the mapped low dimensional space. However, this kind of linear transformation requires many rotations of data from several points of view because the mapping is not a one-to-one. That means the PCA can find a good class separability in its projections when the interesting areas are located along the largest eigenvector the first principal axis. Figure 1 (a) shows an example. On the contrary, the PCA would get a poor separability projection if the areas are not distributed along the first principal axis. Figures 1 (b) and (c) show two different cases. Considering this problem, the PCA is not appropriate to analyze the multispectral images.

The management of multispectral images is difficult compared to one-dimensional (1-D) cases, because there is no total ordering that preserves locality. Once a total ordering is found for a given spatial database, one can use any 1-D access method which may yield good performance for multidimensional data [7], [8]. Thus what is needed is a mapping from a higher dimensional space into a 1-D space. In this mapping, the elements which are in multidimensional

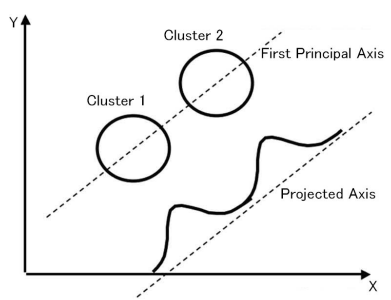

(a)

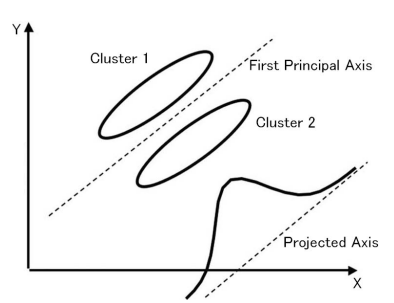

(b)

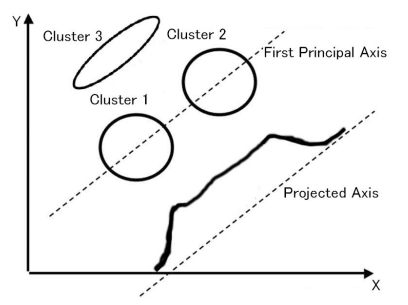

(c)

Fig. 1 Different cases of PCA. 
space are mapped into nearby points on the line to preserve the spatial locality. A space-filling curve is the curve that passes through every point in a multidimensional space and is a one-to-one mapping [1]-[3]. They, especially those curves which have a higher degree of locality, are studied actively in the area of digital image processing as a scan technique because of their properties of preserving the spatial relationship of the multidimensional patterns [4], [16].

In this paper, a new management architecture for multispectral images is proposed. The proposed method utilizes the space-filling curves' properties to map a $N$-dimensional $(N-\mathrm{D})$ space into a $1-\mathrm{D}$ space, so traditional complex multidimensional image clustering can be transformed into a simple one. In our approach, the mapping from $N$-D space to $1-\mathrm{D}$ space is completed by multiple space-filling curves including the Hilbert, $z$ and Pseudo-Hilbert curves. This is because a space-filling curve can give a one-to-one correspondence between the $N$-D coordinates and 1-D sequence numbers, but the obtained 1-D sequence may express wrong relation of points in $N$-D space because of 'Boundary Effects', which is introduced in Sect. 2. In order to overcome this shortcoming, multiple Hilbert curves and $z$ curves generated by shift and rotation, and the Pseudo-Hilbert curve are used together. And to realize the real-time response from the system, data lists storing the group number and 1-D index of data are used. User can obtain the real time response from the system after once making the data lists.

\section{Space-Filling Curves}

A space-filling curve can pass through every point in the $N$ $\mathrm{D}$ space, and is a one-to-one mapping between $N$-D space and 1-D space. Its 1-D order tend to preserve the spacial locality linearly in $N$-D space, this means that points which are close in $N$-D space and represent similar data should be together in the linear 1-D sequence. Sagan [1] discussed a number of space-ordering methods, some examples of which are illustrated in Fig. 1. Each one of them has different characteristics and space-ordering. The raster scan (Fig. 2 (a)), row-prime order (Fig. 2 (b)) and the Cantordiagonal order (Fig. 2 (c)) have a similar scanning way and simple structure. The primary difference between them is only the direction of scan. According to different applications, they are used widely in 2-D image processing. But because of simplicity of their spatial structures, they are very difficult to be used in a $N$-D space. In both $z$ (Fig. 2 (d)) curve and the Hilbert curve (Fig. 2 (e)), the subsquares can be arranged so that the inclusion relationships are preserved, that is, if a square corresponds to an interval, then its subsquares correspond to subintervals of that interval, so these two curves have a higher degree of locality than above three ones. Furthermore they can order a multidimensional space that has been aggregated into hypercubes. Thus the spacefilling curves, as far as we are concerned in this study, are $z$ curve and the Hilbert curve.

Of these two curves, $z$ curve is by far the more frequently used because of its simplicity of the transformation process between the $N$-D data and its corresponding 1-D index. For example, in 2-D case, the 1-D index of a point is obtaining by bit shuffling on the $x$ and $y$ coordinates of the corresponding point in the 2-D space.

Compared to $z$ curve, the Hilbert curve holds a very important property that consecutively ordered points are adjacent in original $N$-D space (if the square Euclidean distance between two points is one, then the two points are called adjacent). In other words, the Hilbert curve can preserve neighboring points as much as possible. The Hilbert curve has two important features which play an important role in those areas benefiting from reducing the dimensionality of the problem:

1. Extracting a group of neighboring pixels having similar

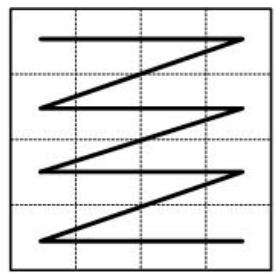

(a)

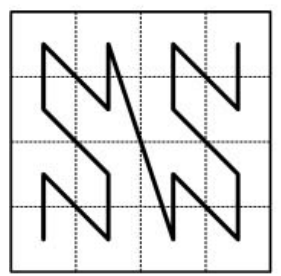

(d)

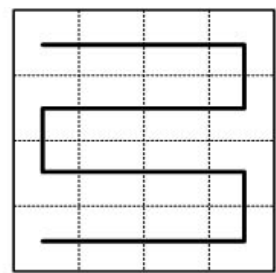

(b)

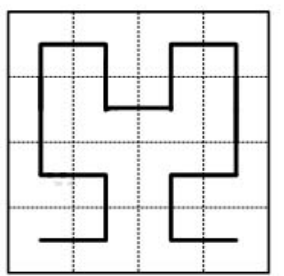

(e)

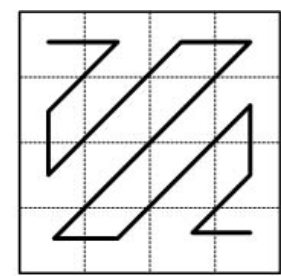

(c)

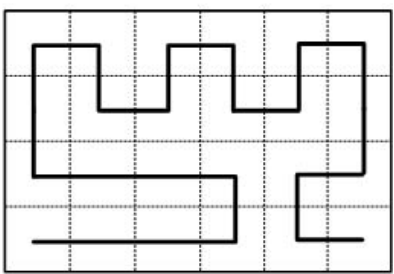

(f)

Fig. 2 Examples of applying a number of different space-filling curves to an $8 \times 8$ image. ((a) Raster scan, (b) Row-prime order, (c) Cantor-diagonal order, (d) $z$ curve, (e) Hilbert curve) (f) Pseudo-Hilbert curve. 
intensities is easier by using the Hilbert scan than other scan techniques.

2. A Hilbert scan can preserve high coherence of neighboring points in an $N$-D space. That is, if scanning length is small, then the corresponding Euclidean distance of multidimensional space is also small.

These two features are quite useful for $N$-D signal processing. Especially, the spatial properties of a $N$-D sequence can be effectively explored in the 1-D space. But note that the converse of hypothesis of the second feature is not true. It may happen that points that are near in $N$-D space are not close along the Hilbert curve. This is because of 'Boundary Effects', which tends to take close $N$-D points far away from each other in certain areas of the Hilbert curve. In fact this problem results from the way how the curve is generated recursively divides $N$-D hypercube into $2^{N}$ sub-hypercubes and each of them is exhaustively traversed before proceeding to the next. Figure 3 shows an example. In the figure, a pair of points $P_{1}$ and $P_{2}$ are neighbors, and the distance between them is 1 . But in the 1-D mapped sequence, the distance becomes to be 21 . This effect will be significantly aggravated with increase of dimensionality.

Based on what we discussed above, we know the Hilbert curve takes advantage of spacial similarities, that is to say, it can get a longer similar sequence with better auto correlation. However, the Hilbert curve also has a constraint that the scanned region must be a square and the length of each side must equal the power of two. So for some applications which don't satisfy this condition, the 1-D sequence expression of a set of $N$-D points may be not correct, that is, 'Boundary Effects' would be enlarged by scanning extra space. To solve this problem, a generalized Hilbert curve the Pseudo-Hilbert curve - is employed in this study. Figure 2 (f) shows a Pseudo-Hilbert curve. In fact, the PseudoHilbert curve is an extension of the Hilbert curve. It has a similar structure as the Hilbert curve and is able to be used for an arbitrarily-sized region. Compared to the Hilbert curve, it provides a much more compact mapping, and this is very useful for avoiding 'Boundary Effect' to some extent. However, the Pseudo-Hilbert curve takes a more expense on computational complexity than the Hilbert curve. So in this study, it is only considered as an assistant of the Hilbert curve to reduce 'Boundary Effects'.

Considering different space-filling curves' own prop-

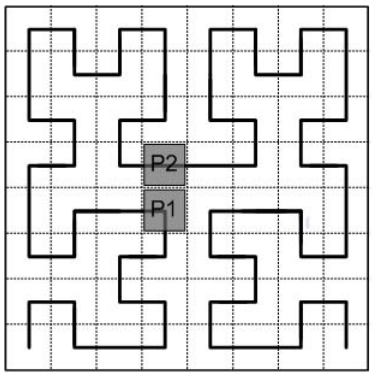

(a)

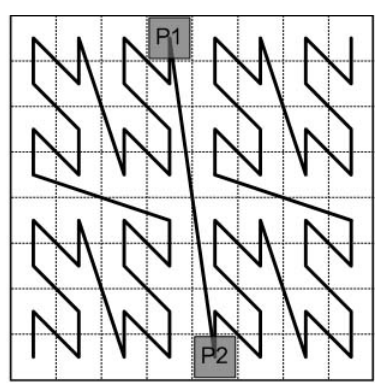

(b)
Fig. 3 Boundary effects. ((a) Hilbert curve, (b) $z$ curve) erties, only one type of space-filling curve is hard to solve 'Boundary Effects' problem. Therefore, the multiple Hilbert curves, $z$ curves, and the Pseudo-Hilbert curve are used together. In the following section, we discuss this in detail.

\section{Cluster Categories by Using Space-Filling Curves}

In this section, a simple clustering algorithm based on the space-filling curves for multispectral images is presented. The proposed approach utilized multiple Hilbert curves, and $z$ curves, and the Pseudo-Hilbert curve to find an exact mapping relation from $N$-D space to $1-\mathrm{D}$ space. Then complicated clustering of multispectral images can be analyzed in a $1-\mathrm{D}$ sequence.

\subsection{1-D Index Assignment Using Space-Filling Curves}

In this paper, an $N$-D Euclidean space with finite granularity is assumed. Thus, we use the $M$-th order approximation of an $N$-D Hilbert curve or $z$ curve $(M \geq 1)$, which maps an $N$-D integer space $\left[0,2^{M}-1\right]^{N}$ into a 1 -D integer set $\left[0,2^{N M}-1\right]$.

Definition 3.1: Let $H_{N}^{M}$ (or $Z_{N}^{M}$ ) denote an $N$-D Hilbert curve (or $z$ curve) with the size of $\underbrace{2^{M} \times \cdots \times 2^{M} \times 2^{M}}_{N}$ which maps the integer space $\left[0,2^{M}-1\right]^{N}$ into $\left[0,2^{M N}-1\right]$. Here $M(M \geq 1)$ is a rank corresponding to the size of the hypercube.

Figures 2 (e) and 3 (a) show two 2-D Hilbert curves corresponding to $H_{2}^{2}$ and $H_{2}^{3}$.

An order tern $\left(X_{1}, X_{2}, \cdots, X_{N}\right)$ of integers $X_{1}, X_{2}, \cdots$, $X_{N}$ is called coordinates of a point. A one-to-one mapping whose domain is a set of $P$ points $(Q)$ and whose image is a set of $P$ non-negative integers $(I)$, is called a scan. If an integer $t(t \in I)$ is the image of a point $\boldsymbol{a}(\boldsymbol{a} \in Q)$ under $f$, i.e. $\boldsymbol{a} \stackrel{f}{\longrightarrow} t$, then $t$ is denoted by $f(\boldsymbol{a})$.

Assuming that there are two $N$-D points $\boldsymbol{a}=$ $\left(X_{1}^{a}, \cdots, X_{N}^{a}\right)$ and $\boldsymbol{b}=\left(X_{1}^{b}, \cdots, X_{N}^{b}\right)$. The square Euclidean distance $d_{u}$ between two points $\boldsymbol{a}$ and $\boldsymbol{b}$ is defined by the following equation

$$
d_{u}=|\boldsymbol{a}-\boldsymbol{b}|^{2}=\left(X_{1}^{a}-X_{1}^{b}\right)^{2}+\cdots+\left(X_{N}^{a}-X_{N}^{b}\right)^{2} .
$$

If $d_{u}$ equals one, then the two points are called adjacent. If $t_{a}$ and $t_{b}$ is the image of $\boldsymbol{a}$ and $\boldsymbol{b}$ in 1-D space respectively, then the scanning length $d_{s}$ between these two points is defined as

$$
d_{s}=|f(\boldsymbol{a})-f(\boldsymbol{b})|=\left|t_{a}-t_{b}\right| .
$$

\subsubsection{Ordering Using the $z$ Curve}

Based on each space-filling curve, any $N$-D point can be characterized by a unique number. In this section, we discuss transformation $f_{z}$ between the 1-D index based the $z$ curve and $N$-D space. As mentioned above, the 1-D index in base 2 (binary number) of the $z$ curve can be easily ob- 
tained by interleaving the bits of $N$-D coordinates in base 2 . So in the first, coordinates of a $N$-D point are transformed into binary form. For example, the coordinates of a point $\left(X_{1}, X_{2}, \cdots, X_{N}\right)$ can be expressed as $N M$-bit binary numbers:

$$
\left\{\begin{array}{c}
X_{1}=x_{1, M} x_{1, M-1} \cdots \cdots x_{1,2} x_{1,1} \\
X_{2}=x_{2, M} x_{2, M-1} \cdots \cdots x_{2,2} x_{1,1} \\
\vdots \quad \vdots \\
X_{N}=x_{N, M} x_{N, M-1} \cdots \cdots x_{N, 2} x_{N, 1},
\end{array}\right.
$$

where $x$ is 0 or 1 . Assuming that the 1-D index of this point in the $Z_{N}^{M}$ can be expressed by an $N M$-bit binary number, i.e.,

$$
\boldsymbol{\alpha}=\underbrace{\overbrace{\alpha_{1, M} \alpha_{2, M} \cdots \alpha_{N, M}}^{N \text { bits }} \cdots \overbrace{\alpha_{1,1} \alpha_{2,1} \cdots \alpha_{N, 1}}^{N \text { bits }}}_{N M \text { bits }} .
$$

And then interleave the bits of $N$-D coordinates in Eq. (2),

$$
\boldsymbol{\alpha}=x_{1, M} x_{2, M} \cdots x_{N, M} \cdots x_{1,1} x_{2,1} \cdots x_{N, 1} .
$$

So the corresponding 1-D index of the point $\left(X_{1}, X_{2}, \cdots\right.$. $X_{N}$ ) can be obtained by transforming the above binary number into a decimal number. Figure 3 (b) shows an example of $2-\mathrm{D}$ case. In this figure, for the lattice point $(2,6)$, its binary form is expressed as follows according to Eq. (2),

$$
\left\{\begin{array}{l}
X_{1}=(2)_{10}=x_{1,3} x_{1,2} x_{1,1}=(010)_{2} \\
X_{2}=(6)_{10}=x_{2,3} x_{2,2} x_{2,1}=(110)_{2}
\end{array}\right.
$$

and its 1-D index is

$$
\boldsymbol{\alpha}=x_{1,3} x_{2,3} x_{1,2} x_{2,2} x_{1,1} x_{2,1}=(011100)_{2}=(28)_{10} .
$$

\subsubsection{Ordering Using the Hilbert and Pseudo-Hilbert Curves}

To obtain the 1-D index from the Hilbert curve, it requires the reflection and rotation recursively on the basic Hilbert curve of 1 st order. In this subsection, we introduce a fast algorithm for generating the Hilbert curve $H_{N}^{M}$. The algorithm traverses an octree with a depth of $m$ using a depth search. The curve from the $m$-th depth nodes to the $(m+1)$ th depth nodes is ordered by the look-up tables [6], where $m=1,2, \cdots, M$. Firstly, we describe the look-up tables for the Hilbert scan. One is a terminal table $\boldsymbol{T}_{\text {trm }}^{N}$ for the address sequences and the other is an induction table $\boldsymbol{T}_{\text {ind }}^{N}$ for the curve type sequences. We use two matrices $-T_{t r m}^{N}[\gamma][i]$ and $T_{\text {ind }}^{N}[\gamma][i]$ - to represent the elements of the two tables, where $1 \leq \gamma \leq N 2^{N-1}$ is the curve type of parent region and $0 \leq i \leq 2^{N}-1$ is an order in each sequence.

Figure 4 shows 2-D basic types of the Hilbert curve. Figure 4 (a) is the case of $\gamma=1$, the address is the first row of the terminal table $\boldsymbol{T}_{\text {trm }}^{2}$ and the order of the child region is the first row of the table $\boldsymbol{T}_{\text {ind }}^{2}$ (Appendix). Figures 4 (b)-(d) are the cases of $\gamma=2,3,4$, respectively in 2-D space.
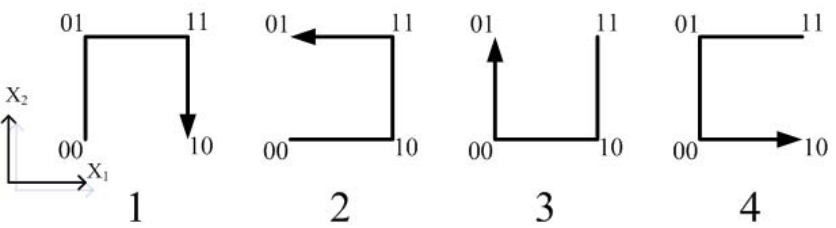

Fig. 4 Basic 2-D Hilbert curve types. (From left to right, figures are arranged from (a), (b), (c) and (d).).

At the first step, coordinates of a point $\left(X_{1}, X_{2}, \cdots, X_{N}\right)$ are also transformed into the binary form shown in Eq. (2). Utilizing $x_{1, M} \cdots x_{N, M}$, we can get the up $N$ bits of the 1-D index in base 2 if $T_{t r m}^{N}[\gamma][i]$ equals $x_{1, M} \cdots x_{N, M}(1 \leq \gamma \leq$ $\left.N 2^{N-1}, 0 \leq i \leq 2^{N}-1\right)$, that is

$$
\alpha_{1, M} \cdots \alpha_{N, M}=(i)_{2}
$$

Here initial value of $\gamma$ equals 1 . Then $\gamma$ is updated by $T_{i n d}^{N}[\gamma][i]$, and the next $N$ bits is obtained in the same way. This is processed recursively until the lowest $N$ bits are made out. And the following algorithm shows this fast Hilbert ordering algorithm generating the 1-D index.

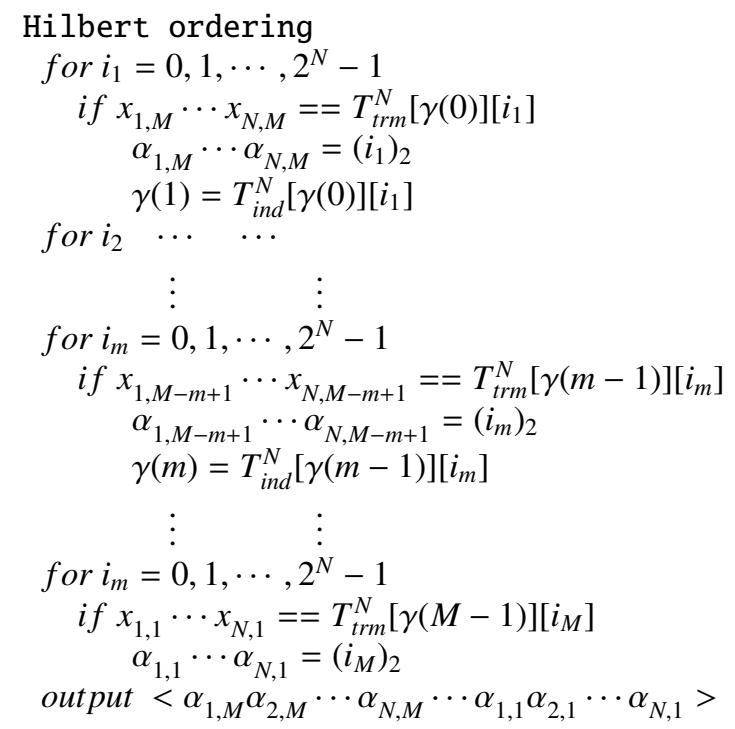

In this algorithm, initial value of $\gamma(0)$ equals one, and $\gamma(m)$ is the curve type at $m$-th division. To explain this mapping relation, an example of 2-D case is shown in Fig. 3 (a). Lattice point $(6,5)$ is firstly transformed into $(111001)_{2}$ by Eq. (3). According to Appendix, we find $T_{t r m}^{2}[1][2]=11$, so $x_{1,3} x_{2,3}=(2)_{10}=(10)_{2}$ and $\gamma$ is updated to $1\left(=T_{\text {ind }}^{2}[1][2]\right)$. In next recursion, because $T_{\text {trm }}^{2}[1][3]$ equals (10) $2, x_{1,2} x_{2,2}$ equals $(11)_{2}\left(=(3)_{10}\right)$ and $\gamma$ is updated to $4\left(=T_{\text {ind }}^{2}[1][3]\right)$. For the lowest 3 bits, $x_{1,3} x_{2,3}$ equals $(01)_{2}\left(=(1)_{10}\right)$ because of $T_{\text {trm }}^{2}$ [4][1] $=01$. Thus the 1-D Hilbert order of the point $(6,5)$ is $(101101)_{2}\left(=(45)_{10}\right)$.

The Pseudo-Hilbert curve is an extension of the Hilbert curve, so it also complies with the Hilbert curve in global sense and holds the property that the 1-D indexes preserve high correlation of the original $N$-D image. Thus its 1- 
D order can be obtained in a similar way which is introduced in the Hilbert scan. However, because it is used for an arbitrarily-sized region, it has a different address assignment. We first define the address assignment of points in a hypercuboid. A hypercuboid with the size $l_{1} \times l_{2} \times \cdots \times l_{N}$ is represented as the following set of points

$$
\begin{aligned}
Q\left(l_{1}, \cdots, l_{N}\right)= & \left\{\left(X_{1}, \cdots, X_{N}\right) \mid 0 \leq X_{1} \leq l_{1}-1,\right. \\
& \left.\cdots, 0 \leq X_{N} \leq l_{N}-1\right\} .
\end{aligned}
$$

Assuming the length of the longest and shortest sides are $l_{\text {max }}$ and $l_{\text {min }}$ respectively, the binary division times of these two sides are obtained by the following equations,

$$
M_{\text {max }}=\left\lfloor\log _{2}\left(\frac{l_{\text {max }}}{2}\right)\right\rfloor, \quad M_{\text {min }}=\left\lfloor\log _{2}\left(\frac{l_{\text {min }}}{2}\right)\right\rfloor
$$

where the operator $\lfloor z\rfloor$ means the integer part of a real number $z$, and the binary division time means how many times the hypercuboid is divided. For each division, each side is divided according to the following rule, which expresses how to divide a side with the length $l$ into two edges with the length: $l(0)$ and $l(1)$.

\section{$\operatorname{division}(l, l(0), l(1))$}

$$
\begin{aligned}
& \text { begin } \\
& m=\left\lfloor\log _{2}\left(\frac{l}{2}\right)\right\rfloor ; \\
& \text { If } 2 \cdot 2^{m} \leq l<3 \cdot 2^{m}, \text { then } l(0) \longleftarrow l-2^{m} \text { and } l(1) \longleftarrow 2^{m} ; \\
& \text { If } l=3 \cdot 2^{m}, \text { then } l(0) \longleftarrow 2 \cdot 2^{m} \text { and } l(1) \longleftarrow 2^{m} ; \\
& \text { If } 3 \cdot 2^{m}<l<4 \cdot 2^{m} \text {, then } l(0) \longleftarrow l-2 \cdot 2^{m} \text { and } \\
& l(1) \longleftarrow 2 \cdot 2^{m} ; \\
& \text { end }
\end{aligned}
$$

Perform above procedure repeatedly for $M_{\min }$ times, each side can be divided into $2^{M_{\min }}$ edges, and the hypercuboid is divided into $\underbrace{2^{M_{N}} \times \cdots \times 2^{M_{N}}}_{N}$ blocks. If the coordinates of $\left(X_{1}, X_{2}, \cdots, X_{N}\right)$ are expressed as $N\left(M_{\max }+2\right)-b i t$ binary numbers like

$$
\left\{\begin{array}{c}
X_{1}=x_{1, M_{\max }+2} x_{1, M_{\max }+1} \cdots \cdots x_{1,2} x_{1,1} \\
X_{2}=x_{2, M_{\max }+2} x_{2, M_{\max }+1} \cdots x_{2,2} x_{1,1} \\
\vdots \vdots \\
\vdots \\
X_{N}=x_{N, M_{\max }+2} x_{N, M_{\max }+1} \cdots \cdots x_{N, 2} x_{N, 1},
\end{array}\right.
$$

where $x_{1, m}, x_{2, m} \cdots$, or $x_{N, m}\left(1 \leq m \leq M_{\max }+2\right)$ are 0 or 1 , the address of a $N$-D point can be expressed by a $N\left(M_{\text {max }}+\right.$ 2) - bit binary number

$$
\underbrace{\overbrace{x_{1, M_{\max }+2} \cdots x_{N, M_{\max }+2} \cdots \overbrace{x_{1,1} \cdots x_{N, 1}}^{N \text { bits }}}^{N \text { bits }}}_{N\left(M_{\max }+2\right) \text { bits }}
$$

The total address of a point consists of two parts - the upper $N M_{\text {min }}$-bit address for the Hilbert address of blocks and the lower $N\left(M_{\max }-M_{\min }+2\right)$-bit address for inner expression of a block. So according to the upper $N M_{\min }$-bit number, we can get the 1-D index $\boldsymbol{\alpha}$ of a block in the Hilbert curve by using Hilbert ordering algorithm introduced above.

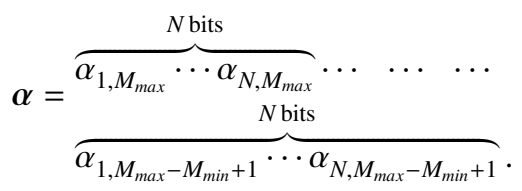

Because the size of each block can be obtained by the division rule, we can calculate number of points in the front $\left((\boldsymbol{\alpha})_{10}-1\right)$ blocks. According to the lower $N\left(M_{\max }-M_{\min }+\right.$ 2)-bit number and $\gamma\left(M_{\min }\right)$, we calculate 1-D index of the point in the current block. At last, the summation of these two parts is the 1-D index of this point in the Pseudo-Hilbert curve (more details can be found in [6]).

From the transformation of Pseudo-Hilbert ordering, we can find that it costs more computation than Hilbert ordering. This is the reason why we don't use directly the Pseudo-Hilbert curve instead of the Hilbert curve.

\subsection{Clustering Categories for Multispectral Images}

Firstly, we assume that $T$ multispectral images are represented as $I_{1}, I_{2}, \cdots I_{T}$, whose resolution is $\mu_{\max } \times v_{\max }$. A pixel $(\mu, v)\left(0 \leq \mu \leq \mu_{\text {max }}-1,0 \leq v \leq v_{\text {max }}-1\right)$ has an intensity value in each band image, so after combining $N$ intensity values together, the content of the pixel $(\mu, v)$ can be represented as a $N$-D vector $\left(I_{1}(\mu, v), \cdots, I_{N}(\mu, v)\right)$. In fact this vector can be looked as coordinates of a point in a $T$-D space, that is

$$
\left(X_{1}, X_{2}, \cdots, X_{T}\right)=\left(I_{1}(\mu, v), I_{2}(\mu, v), \cdots, I_{T}(\mu, v)\right)
$$

Based on what we discussed in Sect. 3.1, we know that the biggest problem about application of the Hilbert curve is its 'Boundary Effects'. In this study, we use multiple Hilbert curves, and $z$ curves, and the Pseudo-Hilbert curve to solve it. Here, multiple Hilbert curves are generated by shifting all data in different direction. For example, in Fig. 3 (a), we shift $P_{1}$ and $P_{2}$ one unit down

$$
\begin{aligned}
& P_{1}=(3,3-1)=(3,2), \\
& P_{2}=(3,4-1)=(3,3),
\end{aligned}
$$

then according to the Hilbert ordering algorithm, we can get new Hilbert orders for these two points as follows.

$$
\begin{aligned}
& \boldsymbol{\alpha}_{p_{1}}=(001001)_{2}=(9)_{10}, \\
& \boldsymbol{\alpha}_{p_{2}}=(001010)_{2}=(10)_{10} .
\end{aligned}
$$

After shifting, $P_{1}$ and $P_{2}$ are now adjacent in Hilbert order, which preserves the real relation in 2-D space and avoids boundary effects effectively. So as to points with different locations in $T$-D space, if we want to find their true relation from 1-D Hilbert orders, the more times we shift these points in different directions, the more accurate relation we are more likely to get. 
If the intensity of each band image is less than $2^{M}, H_{T}^{M}$ is used to order multispectral images. The number of pixels is $\mu_{\max } \times v_{\max }$. So we can calculate pixel distribution in $T$-D intensity space. Based on the pixel distribution characteristics, we can identify different categories. In other words, when the number of pixels is high in some area of $T$-D space, these pixels can be recognized as a same category. So in order to find out the area whose $T$-D intensity values are adjacent or close, we first use multiple Hilbert curves to order these $T$-D data. In this study, considering the computational complexity, we shift the data no more than 2 units, that is, for each direction $\nabla X_{n}(1 \leq n \leq T)$ is in a range from -2 to 2 . So total shifting times are $4^{T}$. At first, we introduce that how to calculate $H L_{0}$ which has no shift. For each $T$-D intensity point, we use the proposed method based on the look-up tables (Sect. 3.1.2) to get 1-D Hilbert index. Then these data are arranged in accordance with the Hilbert order, and the data which are adjacent are classified into a group. So the order and group information are record in $H L_{0}$. Table 1 shows an example of $H L_{0}$. In Table 1 , the 1-D location of a pixel is record in a order from left to right and top to down. For instance, the 1-D location of pixel $(\mu, v)$ is obtained by $\mu v_{\max }+v$. Group number start from 1 to $\mathrm{K}$, and it is decided once the arrangement of Hilbert ordering is finished in $H L_{0}$. Then for each time, after shifting all the data, we can calculate the new Hilbert order in the same way. Finally, we can get other $4^{T}-1$ Hilbert-sorted lists $H L_{1}, H L_{2}, \cdots, H L_{4^{T}-1}$. Table 2 shows an example of $H L_{i}\left(1 \leq i \leq 4^{T}-1\right)$. Thus using the obtained new Hilbert orders, we rearrange the data stored in $H L_{0}$ recursively until we get $H L_{0}\left(4^{T}-1\right)$, where $H L_{0}(i)\left(1 \leq i \leq 4^{T}-1\right)$ means $H L_{0}$ is updated for the $i$-th time.

$$
\begin{aligned}
& \text { Update of Hilbert-ordered list } \\
& \text { for } i=1, \cdots, 4^{T}-1 \\
& \text { for } k=1, \cdots, K \\
& \text { In } H L_{i}: \\
& \text { Search the Groups whose distance is less } \\
& \text { than } d_{s} \text { from Group } k \\
& \text { In } H L_{0}(i): \\
& \text { Put found Groups together with Group } k \\
& \text { In } H L_{0}(i):
\end{aligned}
$$

Table 1 The Hilbert-sorted list $H L_{0}$.

\begin{tabular}{llll}
\hline Group & $(\boldsymbol{\alpha})_{10}$ & Pixel's location & Number of pixels \\
\hline 1 & 38 & $15,16,18,19,245,246$ & 6 \\
2 & 41 & $34,35,224,225,227$ & 5 \\
$\ldots$ & $\ldots$ & $\ldots$ & $\ldots$ \\
\hline
\end{tabular}

Table 2 The Hilbert-sorted list $H L_{i}$.

\begin{tabular}{llll}
\hline Group & $(\boldsymbol{\alpha})_{10}$ & Pixel's location & Number of pixels \\
\hline 5 & 25 & $21,22,23,1134,1135$ & 5 \\
3 & 29 & $30,31,415$ & 3 \\
$\ldots$ & $\ldots$ & $\ldots$ & $\ldots$ \\
\hline
\end{tabular}

\section{Merge groups which have same Group number}

In the above algorithm, the distance between Group $k 1$ and $k 2$ in a same Hilbert-sorted list is calculated by

$$
\left|(\boldsymbol{\alpha})_{10}\right|_{k 1}-\left.(\boldsymbol{\alpha})_{10}\right|_{k 2} \mid
$$

where $(\boldsymbol{\alpha})_{10} \mathrm{l}_{k}$ means Group $k$ 's 1-D Hilbert index. $d_{s}$ can be set in accordance with user's requirement. When $d_{s}$ is a small value, multispectral images are able to be analyzed in a fine data distribution. On the contrary, we can analyze multispectral images in a coarser data distribution when $d_{s}$ become bigger.

Based on the discussion of Sect.2, we know that 'Boundary Effects' would become worse if the T-D multispectral space is smaller than the used Hilbert curve. For example, assuming that the size of a scanned region is $8 \times 6$. In Fig. 5 (a), we scan this region by using the Hilbert curve $H_{2}^{3}$, then we can find that among three points $P 1, P 2$ and $P 3$, the scanning length between $P 1$ and $P 3$ is smallest. However, this is wrong to describe relations of these three points in the original 2-D space, the reason of which is because 'Boundary Effects' happens between $P 1$ and $P 2$ and moreover this effect is enlarged by scanning extra up two rows. Thus in order to remove this constraint and provide a right 1-D scanning length corresponding to Euclidean distance of $T$-D multispectral space, the Pseudo-Hilbert scan is used to revise the obtained $H L_{0}\left(4^{T}-1\right)$. Thus firstly, a PseudoHilbert-ordered list $P H L$ is made in a similar way as $H L$. Then $H L_{0}\left(4^{T}-1\right)$ is updated to $H L_{0}\left(4^{T}\right)$ in the light of PHL. From Fig. 5 (b), we can see that the scanning length between $P 1$ and $P 2$ can be corrected through the PseudoHilbert scan.

A $T$-D data has $3^{T}-1$ neighbors, and generally we hope these data belong to a same group. Thus to let the points located at the diagonal line has a same distance as the ones which are adjacent, we use multiple $z$ curves to reduce the distance. Multiple $z$ curves are generated by exchanging the coordinates. So for a $Z_{T}^{m}$ curve, there are $T$ ! forms. For example, if we rotate the curve of Fig. 3 (b) $90^{\circ}$ in a clockwise direction, the new $z$ order is shown in Fig. 6. Here note that because the $z$ curve is not a continuous curve, 'Boundary Effects' also exists in some certain regions. However, 'Boundary Effects' of the $z$ curve is not same as the Hilbert

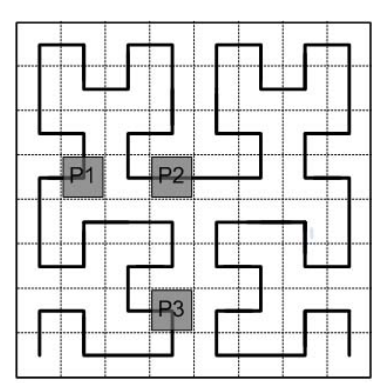

(a)

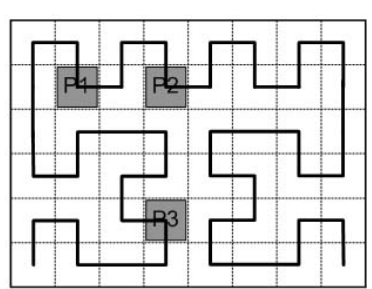

(b)
Fig. 5 Scan a $8 \times 6$ region with the Hilbert curve and Pseudo-Hilbert curve. 
curve's. In fact, it is contrary, that is to say, the $z$ curve may take $T$-D points which are far away very close to each other in certain areas of the Hilbert curve. Thus after we construct a $z$-sorted list $Z L_{0}$ in the way which is used in the Hilbertsorted list, in each group, we should make a judgement that whether the points of this group belongs to a same interval $[4 \times k, 4 \times k+3]\left(0 \leq k \leq 2^{T m-2}-1\right)$. If there is a point which does not satisfy this condition, this point is considered as a false connection and this group will be divided into two groups. So 'Boundary Effects' can be removed. In order to demonstrate this property, we compared the proposed multiple scans with the single Hilbert scan using a statistical method [6]. Figure 7 is the case with the size $64 \times 64$. From the figure, we can see that both of curves are similar, but the blue curve is much smoother than the other blue one, especially in the range with small square Euclidean distance $(<800)$. This means that the scanning length obtained by multiple scans is almost proportional to the square Euclidean distance, which proves that the obtained 1-D data could preserve the high correlation of multidimesional data without 'Boundary Effects'.

At last, update $H L_{0}\left(4^{T}\right)$ by using $Z L_{0}(T !-1)$, we can get a final order of 1-D data. For multispectral images, based on this 1-D sequence, a pixel belongs to a class or not depending on its scanning length without calculating $T$-D Euclidean distance or using any texture information.

\section{Experimental Results}

Among so many clustering algorithms, the $k$-means algo-

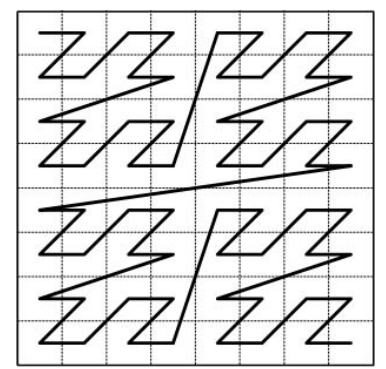

Fig. 6 The $z$ curve after rotation 90 degree.

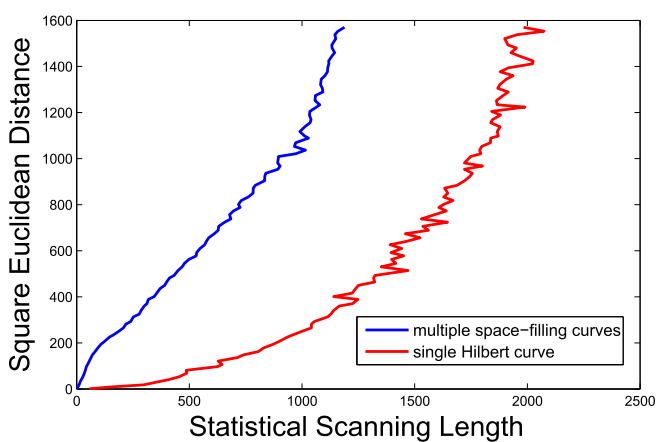

Fig. 7 Relation between square Euclidean distance and the statical scanning length between two points.

rithm [11] which is also based on distance-based clustering, and the fuzzy $c$-means algorithm [18], [19] are probably the most widely applied clustering approaches. But they also has some drawbacks in implementation, for example, for the $k$-means algorithm, it is difficult to determine the parameter $k$ to represent "natural" cluster, and it is only suitable for concave spherical clusters. In Fig. 8, the left two figures show the clustering results obtained by the $k$-means algorithm. In these two cases, the $k$-means approach cannot get right clusters. As a comparison, we show the results obtained by the proposed algorithm in Fig. 8 (right two figures). From the figures, it can be seen clearly that the proposed algorithm discover true clusters for these special shapes without complex computation. This property may be very efficient even for large spatial databases. Both of the $k$-means and fuzzy $c$-means algorithms have high computational complexity, and this makes them difficult to implement in large databases. In Table 3, a comparison of the execution time is made for different dimensional conditions. Here assuming that the number of clusters is five, and number of data points is $512 \times 400$. From the table, we can find that the fuzzy $c$-means algorithm takes the most time to cluster among these three methods. Compared to the $k$ means algorithm, the proposed algorithm have a lower time cost in 3-D and 5-D cases, while in 7-D case it takes more time. Because more Hilbert-sorted lists should be calculated with increase of dimensions, the time cost of the proposed algorithm always increases. Thus it could be inferred that in $N$-D $(N>7)$ space, our approach will take more time to cluster. However, this won't effect its efficiency when we use it for multi-spectral images. Because once the number of dimensions is fixed, the time cost of the proposed algo-
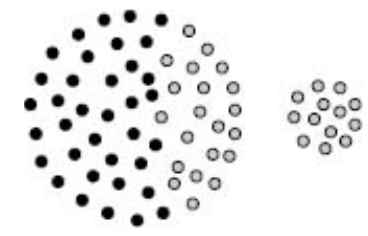

(a)

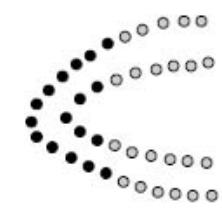

(a)

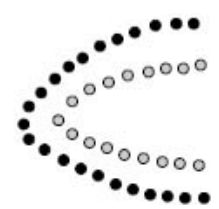

(b)

Fig. 8 Cluster treated with different algorithms, left: the $k$-means method; right: proposed method.

Table 3 A comparison of the execution time under different dimensions (assuming the number of clusters is five).

\begin{tabular}{llll}
\hline $\begin{array}{l}\text { Number of } \\
\text { dimensions }\end{array}$ & $k$-means & Fuzzy & $\begin{array}{l}\text { our approach } \\
\text { (build) }\end{array}$ \\
\hline 3 & $21.312(\mathrm{~s})$ & $563.6430(\mathrm{~s})$ & $1.245(\mathrm{~s})$ \\
5 & $12.356(\mathrm{~s})$ & $383.834(\mathrm{~s})$ & $7.844(\mathrm{~s})$ \\
7 & $18.570(\mathrm{~s})$ & $405.3830(\mathrm{~s})$ & $35.844(\mathrm{~s})$ \\
\hline
\end{tabular}


rithm does not change so much when the number of cluster increases. But the $k$-means algorithm' time cost would increase greatly if the number of clusters become big. Table 4 illustrate this.

In this paper, a 7-D landsat TM image is used to test the performance of our algorithm. It's shown in false color with bands 1, 2, and 3 mapped to R, G, and B planes respectively, in Fig. 10 (a) (for displaying purposes only, the intensity in each band is stretched to achieve high contrast). At the first step, the TM image with seven bands are loaded

Table 4 A comparison of the execution time (assuming the number of dimensions is five).

\begin{tabular}{lll}
\hline Number of clusters & $k$-means & our approach \\
\hline 15 & $117.424(\mathrm{~s})$ & $9.423(\mathrm{~s})$ \\
30 & $419.513(\mathrm{~s})$ & $10.688(\mathrm{~s})$ \\
60 & $845.223(\mathrm{~s})$ & $12.208(\mathrm{~s})$ \\
\hline
\end{tabular}

from database, and a 7-D vector for content of each pixel is constructed by Eq. (11). Then by the method introduced in Sect. 3.1, the 7-D data are transformed several 1-D sequences. These 1-D data are arranged according to different space-filling curves' orders, and lists $(H L, P H L, Z L)$ are generated. Finally, by adjusting the parameter $d_{s}$, the data list can be updated and different categories are identified from different views according to user's requirement.

In our experiments, from coarse data distribution to fine data distribution, 3, 5, 9, 14, and 512 categories are extracted by the proposed algorithm. Figure 9 shows data distributions corresponding to 512,14 , and 5 categories. The classification maps is shown in Figs. 10 (b)-(f) corresponding to $512,14,9,5$, and 3 categories respectively. The resultant classified regions include the classes: mountain, coast, water, artificial construction, downtown urban region, suburban urban region, and mixed agricultural fields and so on. From our experiments, we can see that the proposed ap-
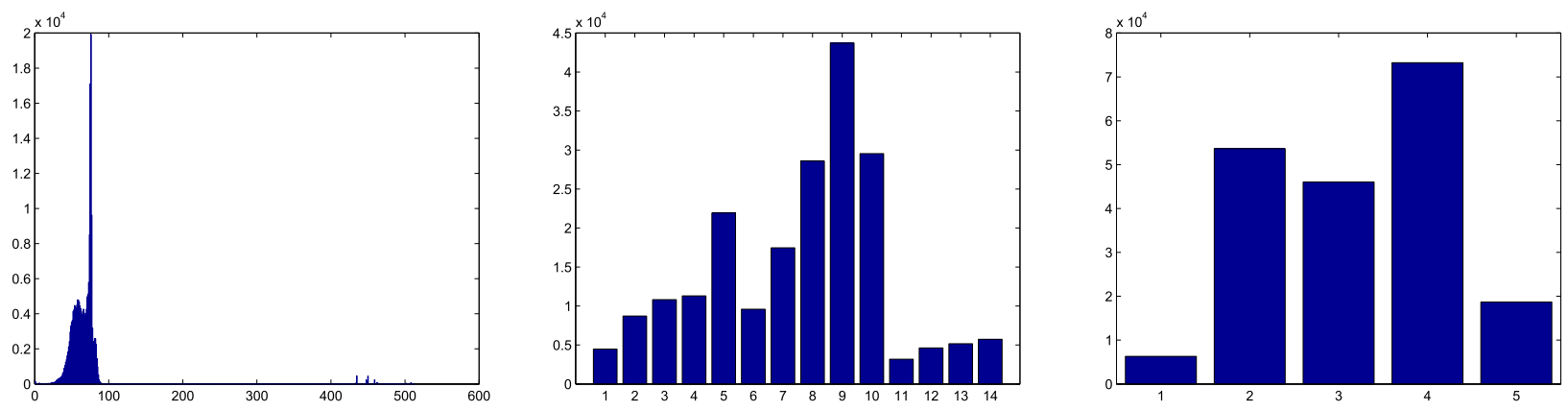

Fig. 9 Data distributions corresponding to 512, 14, and 5 categories (left to right).
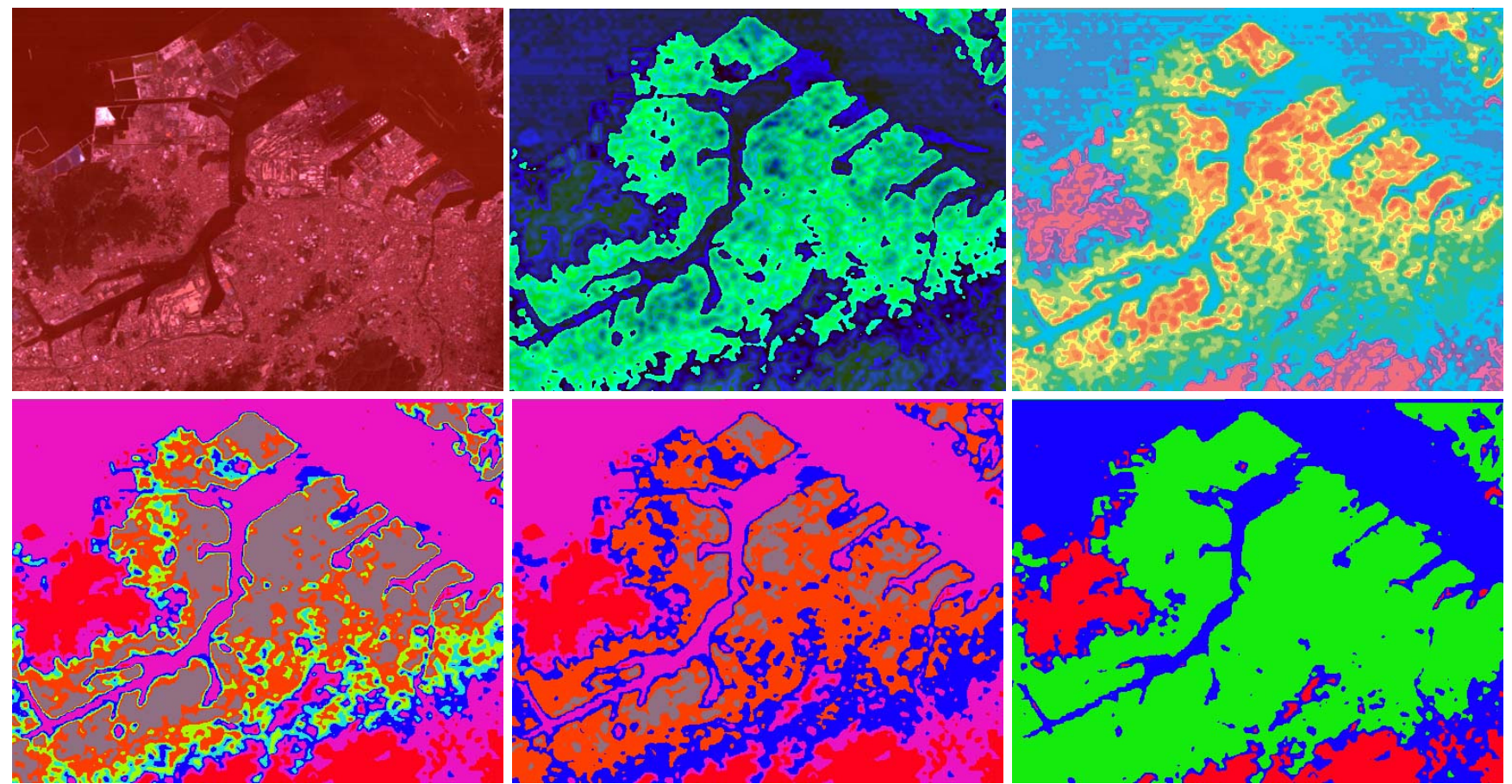

Fig. 10 A landsat TM image (a) and the classified regions of it (b)-(f) (figures' order is from left to right, top to bottom. 
proach doesn't calculate $N$-D Euclidean distance, or use any texture information, or even some complex transformation, but only use its 1-D scanning distance $d_{s}$ to be able to cluster the multidimensional data. This demonstrates efficiency of the proposed method.

\section{Conclusion}

In this paper, we propose a simple method for management of multispectral images by using space-filling curves. This approach suggests that the clustering of multispectral images can be analyzed by multiple Hilbert curves, $z$ curves, and the Pseudo-Hilbert curve, which set up a one-to-one mapping relationship between a $N$-D space and 1-D space. Therefore, the proposed method extracts categories from 1$\mathrm{D}$ data mapped by these curves without computing any distance in $N$-D space. Furthermore, multispectral images can be analyzed hierarchically from coarse data distribution to fine data distribution in accordance with different application. The experimental results performed on LANDSAT data have demonstrated that the proposed method is efficient to manage the multidimensional images and can be applied easily.

\section{References}

[1] H. Sagan, Space-filling Curve, Springer-Verlag, 1994.

[2] A.R. Butz, "Space filling curves and mathematical programming," IEEE Trans. Information and Control, vol.12, no.4, pp.314-330, 1968.

[3] A.R. Butz, "Convergence with Hilbert's space filling curve," IEEE J. Computer and System Sciences, vol.3, no.2, pp.128-146, 1969.

[4] S. Kamata, M. Niimi, and E. Kawaguchi, "Interactive analysis of multispectral images using a Hilbert curve," Proc. 12th IEEE Int. Conf. Pattern Recognition, pp.93-97, 1994.

[5] H. Samet, The Design and Analysis of Spacial Data Structures, Addison-Wesley, 1990.

[6] J. Zhang and S. Kamata, "An N-dimensional pseudo-Hilbert scan for arbitrarily-sized hypercuboids," IEICE Trans. Fundamentals, vol.E91-A, no.3, pp.846-858, March 2008.

[7] B. Moon, H.V. Jagadish, C. Faloutsos, and J.H. Saltz, "Analysis of the clustering properties of the Hilbert spacefilly curve," IEEE Trans. Knowledge Data Eng., vol.E91-A, no.3, pp.124-141, 2001.

[8] D. Hilbert, "Uber die stetige abbidung einer linie auf flachenstuck," Math. Annln., vol.38, pp.459-460, 1981.

[9] G.A. Carpenter, S. Grossberg, N. Markuzon, J.H. Reynolds, and D.B. Rosen, "Fuzzy ARTMAP: A neural network architecture for incremental supervised learning of analog multidimensional maps," IEEE Trans. Neural Netw., vol.3, pp.698-713, 1992.

[10] G. Fung, A Comprehensive Overview of Basic Clustering Algorithms, 1992.

[11] T. Kanungo, D.M. Mount, N.S. Netanyahu, C.D. Piatko, R. Silverman, and Y. Wu, "An efficient $k$-means clustering algorithm: Analysis and implementation," IEEE Trans. Pattern Anal. Mach. Intell., vol.24, no.7, pp.881-892, 2002.

[12] J.C. Bezdek, Pattern Recognition with Fuzzy Objective Function Algorithms, Plenum Press, New York, 1992.

[13] F. Salzenstein and C. Collet, "Fuzzy Markov random fields versus chanins for multispectral image segmentation," IEEE Trans. Pattern Anal. Mach. Intell., vol.28, pp.1753-1767, 2006.

[14] J. Goldberger and S. Roweis "Hierarchical clustering of a mixture model," NIPS, 2004.
[15] P.J. Toivanen, J. Ansamaki, J. Parkkinen, and J. Mielikainen, "Edge detection in multispectral images using the self-organizing map," Pattern Recognit. Lett., vol.24, pp.2987-2994, 2003.

[16] L.O. Jimenez and D.A. Landgrebe, "Supervised classification in high dimentsional space: Geometrical, statistical, and asymptotical properties of multivariate data," IEEE Trans. Syst., Man, Cybern. C, Appl. Rev., vol.28, no.1, pp.39-54, 1998.

[17] J.A. Richards, "Remote sensing digital image analysis," in An Introduction, 3rd ed., Springer-Verlag, New York, 1999.

[18] N.R. Pal and J.C. Bezdek, "On cluster validity for the fuzzy c-means model," IEEE Trans. Fuzzy Systems, vol.3, pp.370-379, 1995.

[19] J.C. Bezdek, R. Ehrlich, and W. Full, "FCM: The fuzzy c-means clsutering algorithm," Computers \& Geosciences, vol.10, pp.191203, 1984 .

\section{Appendix: Two-Dimensional Look-Up Tables}

In 2-D space, Hilbert address sequences (Terminal table) and curve type sequences (Induction table) are shown as follows.

$$
\begin{aligned}
& \boldsymbol{T}_{\text {trm }}^{2}=\left[T_{\text {trm }}^{2}[\gamma][i]\right]=\left[\begin{array}{llll}
00 & 01 & 11 & 10 \\
00 & 10 & 11 & 01 \\
11 & 10 & 00 & 01 \\
11 & 01 & 00 & 10
\end{array}\right], \\
& \boldsymbol{T}_{\text {ind }}^{2}=\left[T_{\text {ind }}^{2}[\gamma][i]\right]=\left[\begin{array}{llll}
2 & 1 & 1 & 4 \\
1 & 2 & 2 & 3 \\
4 & 3 & 3 & 2 \\
3 & 4 & 4 & 1
\end{array}\right]
\end{aligned}
$$

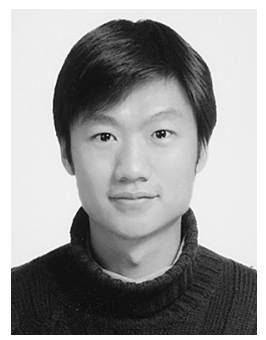

Jian Zhang received the B.E. in electrical engineering from Nanjing University of Information Science and Technology, Nanjing, China, in 2003, the M.E. degree in communication and information Engineering from Shanghai University, Shanghai, China, in 2005, and the second M.E. degree and Ph.D. degree in computer science from Waseda University, Fukuoka, Japan, in 2006 and 2010. He is now a researcher at Information, Production and Systems Research Center, Waseda University, Fukuoka, Japan. His current research is main on the space-filling curve application, pattern recognition and image processing.

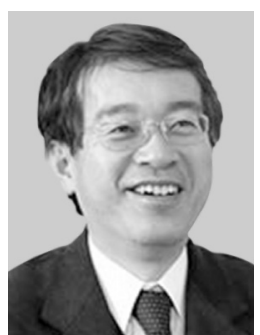

Sei-ichiro Kamata received the M.S. degree in computer science from Kyushu University, Fukuoka, Japan, in 1985, and the Doctor of Computer Science, Kyushu Institute of Technology, Kitakyushu, Japan, in 1995. From 1985 to 1988, he was with NEC, Ltd., Kawasaki, Japan. In 1988, he joined the faculty at Kyushu Institute of Technology. From 1996 to 2001, he has been an associate professor in the Department of Intelligent System, Graduate School of Information Science and Electrical Engineering, Kyushu University. Since 2003, he has been a professor in the Graduate School of Information, Production and Systems, Waseda University. In 1990 and 1994, he was a Visiting Researcher at the University of Maine, Orono. His research interests include image processing, pattern recognition, image compression, and space-filling curve application. Dr. Kamata is a member of the IEEE and the ITE in Japan. 